\title{
Genome Sequence of the Plant Growth-Promoting Rhizobacterium Bacillus sp. Strain 916
}

\author{
Xiaoyu Wang, Chuping Luo, and Zhiyi Chen \\ Institute of Plant Protection, Jiangsu Academy of Agricultural Sciences, Nanjing, China
}

Bacillus sp. strain 916, isolated from the soil, showed strong activity against Rhizoctonia solani. Here, we present the high-quality draft genome sequence of Bacillus sp. strain 916. Its 3.9-Mb genome reveals a number of genes whose products are possibly involved in promotion of plant growth or antibiosis.

R ice is the largest crop in Asia. However, fungal diseases have severely affected rice production and quality. Rhizoctonia solani causes rice sheath blight in rice, which is a devastating disease because of its extensive damage to rice yield and quality. Bacillus sp. strain 916, isolated from soil, showed strong activity against $R$. solani and served as an important biocontrol agent in rice disease by its ability to promote plant growth and to suppress plantpathogenic organisms $(3,4)$. The Bacillus sp. strain 916 product has been extensively field-tested and registered as a biofungicide. It was used on 3.33 million hectares of rice in $2011(8,10)$. The 16S rRNA gene sequences of strain 916 are closely related to those of Bacillus subtillis 168 (99.6\%) and Bacillus amyloliquefaciens subsp. plantarum FZB42 (99.9\%).

The genome sequencing was performed with Illumina/Solexa HiSeq 2000 at BGI in China. Sequence reads were generated from a 475-bp paired-end library. Total paired reads were de novo assembled with SOAPdenovo software (BGI; http://soap.genomics .org.cn/soapdenovo.html), an in-house assembler based on the de Bruijin graph theory. Protein-encoding genes, rRNA operons, and tRNAs were predicted by Glimmer (5), RNAmmer (7), and tRNAscan (13), respectively. Annotation was performed using the GenBank, COG, Pfam, and TIGRFam databases and the RAST server (1).

The final assembly consists of a single contig of 3,925,928 bp with $46.64 \% \mathrm{G}+\mathrm{C}$ content. A total of 2,814 protein-coding sequences were assigned predicted functions. The genome has 39 rRNA operons and 46 tRNAs. Bacillus sp. strain 916 harbors eight giant gene clusters directing synthesis of bioactive peptides and polyketides by modularly organized megaenzymes named nonribosomal peptide synthetases (NRPS) and polyketide synthases (PKS), such as srf, bmy, and fen $(11,12)$. Besides these three sets, there are four gene clusters for biosynthesis of nonribosomal peptides and polyketides that may function as antibiotics, similar to bacilysin, difficidin, bacillaene, and macrolactin, and for synthesis and transport of bacillibactin, a high-affinity siderophore that may inhibit the growth of fungal pathogens $(2,9)$. Macrolactin, difficidin, and bacillomycin L ( bmyCBAD gene cluster) are absent from Bacillus subtilis 168 strains. More than $8.01 \%$ of the genome is devoted to synthesizing antibiotics and siderophores by pathways not involving ribosomes. Bacillus sp. strain 916 also contains a phytase gene, comAPQX, and $s f p$, related to the biocontrol mechanism. Idriss et al. report that the extracellular phytase activity of Bacillus amyloliquefaciens FZB45 contributes to its plant growthpromoting effect (6); our previous study demonstrated that the mutation of three key amino acids greatly affected the biological activity of $\operatorname{com} A$, which caused reductions in competence, spore formation, and surfactin production in Bacillus sp. strain 916 (14). The Bacillus sp. strain 916 genome contains many gene clusters encoding a variety of antimicrobial substances; it can be considered a paradigm for a unique group of plant-associated Grampositive bacteria with huge potential for biocontrol and plant growth promotion. The complete genome sequence, along with its amenability to genetic manipulation, should facilitate exploitation of the hitherto unappreciated potential of Bacillus sp. strain 916 to produce secondary metabolites for development of agrobiological engineering preparations.

Nucleotide sequence accession numbers. The draft genome sequence has been deposited in GenBank under accession no. AFSU00000000 (first version, AFSU01000000).

\section{ACKNOWLEDGMENTS}

We thank Lijun Ma for critical comments on the manuscript.

This work was supported by the National Natural Science Foundation of China (grants no. 30971950 and 30900929 ).

\section{REFERENCES}

1. Aziz R, et al. 2008. The RAST Server: rapid annotations using subsystems technology. BMC Genomics 9:75. doi:10.1186/1471-21649-75.

2. Butcher RA, et al. 2007. The identification of bacillaene, the product of the PksX megacomplex in Bacillus subtilis. Proc. Natl. Acad. Sci. U. S. A. 104:1506-1509.

3. Chen ZY, Xu ZG, Lu F, Liu YF, Chen YL. 2000. On antagonism against Rhizoctonia solani of culture solution of strain B-916 and constituent of its antifungal substance. Jiangsu J. Agric. Sci. 16:148-152. (In Chinese.)

4. Chen ZY, Xu ZG, Lu F, Liu YF. 2001. The inducing resistance effect of antagonistic bacterium B-916 on rice plant. Southwest China J. Agric. Sci. 14:44-48. (In Chinese.)

5. Delcher AL, Bratke KA, Powers EC, Salzberg SL. 2007. Identifying bacterial genes and endosymbiont DNA with Glimmer. Bioinformatics 23:673-679.

6. Idriss EE, et al. 2002. Extracellular phytase activity of Bacillus amyloliquefaciens FZB45 contributes to its plant-growth-promoting effect. Microbiology 148:2097-2109.

Received 13 July 2012 Accepted 17 July 2012

Address correspondence to Xiaoyu Wang,wxyz999@126.com, or Zhiyi Chen, chzy@jaas.ac.cn.

Copyright @ 2012, American Society for Microbiology. All Rights Reserved. doi:10.1128/JB.01266-12 
7. Lagesen K, et al. 2007. RNAmmer: consistent and rapid annotation of ribosomal RNA genes. Nucleic Acids Res. 35:3100-3108.

8. Li DQ, Nie YF, Wei LH, Wei BQ, Chen ZY. 2007. Screening of highyielding biocontrol bacterium Bs-916 mutant by ion implantation. Appl. Microbiol. Biotechnol. 75:1401-1408.

9. Lugtenberg B, Kamilova F. 2009. Plant-growth-promoting rhizobacteria. Annu. Rev. Microbiol. 63:541-556.

10. Luo CP, et al. 2010. The operon, structure and biological activities of the lipopeptide bacillomycin L produced by Bacillus subtilis Bs916. Sci. Agric. Sin. 43:4624-4634. (In Chinese.)

11. Nakano MM, et al. 1991. srfA is an operon required for surfactin produc- tion, competence development, and efficient sporulation in Bacillus subtilis. J. Bacteriol. 173:1770-1778.

12. Ongena M, Jacques P. 2008. Bacillus lipopeptides: versatile weapons for plant disease biocontrol. Trends Microbiol. 16:115-125.

13. Schattner P, Brooks AN, Lowe TM. 2005. The tRNAscan-SE, snoscan and snoGPS web servers for the detection of tRNAs and snoRNAs. Nucleic Acids Res. 33:W686-W689.

14. Wang XY, et al. 2010. Three non-aspartate amino acid mutations in the ComA response regulator receiver motif severely decrease surfactin production, competence development and spore formation in Bacillus subtilis. J. Microbiol. Biotechnol. 20:301-310. 